\title{
Italique
}

Poésie italienne de la Renaissance

XX $\mid 2017$

Varia

\section{Diletti comico-bucolici o della maniera rustica tra scena e tipografia}

Chiara Lastraioli

\section{(2) OpenEdition}

Journals

Edizione digitale

URL: http://journals.openedition.org/italique/463

DOI: $10.4000 /$ italique.463

ISSN: 1663-4438

Editore

Librairie Droz

\section{Edizione cartacea}

Data di pubblicazione: 1 ottobre 2017

Paginazione: 195-210

ISBN: 978-2-600-05818-6

ISSN: 1423-3983

Notizia bibliografica digitale

Chiara Lastraioli, «Diletti comico-bucolici o della maniera rustica tra scena e tipografia », Italique

[Online], XX | 2017, online dal 01 octobre 2019, consultato il 23 janvier 2020. URL : http://

journals.openedition.org/italique/463 ; DOI : 10.4000/italique.463

(c) Tous droits réservés 
Chiara LASTRAIOLI

\section{I L E T T I C O M I C O - B U C O L I C I \\ O DELLA MANIERA RUSTICA \\ TRA SCENA E TIPOGRAFIA}





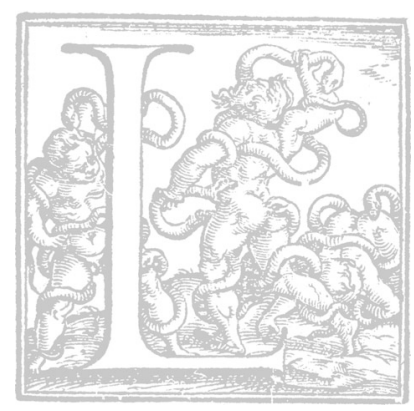

'incursione di tematiche e stilemi bucolici nella letteratura comico-burlesca del Cinquecento è un dato ormai assodato, sebbene esso resti poco studiato dagli specialisti dei generi letterari. Da qualche anno indago, con l'aiuto di pazienti e tenaci collaboratori, $i$ destini dell'edizione cinquecentesca in lingua italiana nel mondo francofono della prima età moderna, il che mi ha allontanato dalle raffinate disanime dei testi lirici e colti e, in parte, dalla letteratura che mi è più congeniale, cioè quella satirica e di propaganda. Tuttavia, la pratica di numerosi fondi librari transalpini mi ha permesso di vagliare, in modo per ora eminentemente quantitativo, la consistenza della produzione comico-bucolica e di trarne alcune conclusioni quanto alle tipologie testuali e editoriali.

Tra gli autori della prima generazione del cosiddetto "Rinascimento capriccioso e irregolare", ' quella del Berni, dell'Aretino, del Bentivoglio, del Caccia per intenderci, non pochi furono coloro che praticarono, con esiti talvolta non del tutto indegni, la scrittura bucolica. Per l'Aretino della giovanile Opera nova, che data del I5I2, ${ }^{2}$ la sola egloga dell'intera raccolta è il necessario tributo di un aspirante poeta cortigiano che tenta di imporsi quale poliedrico poeta all'improvviso. Sin dalla dedica al «lepidissimo lettore», in cui si offrono «cose da noi facte in uno quasi istante», per cui «si non l'opra al manco l'audacia piacerà), '3 l'autore manifesta l'intenzione di sfruttare la possente scia tracciata dall'A quilano prima, e da Bernardo Accolti poi, quale trampolino di lancio verso una carriera di poeta curiale ancora tutta da costruire. ${ }^{4} \mathrm{Il}$ "carattere arcaico" dell'organizzazione del volumetto, che riprende il modello quattrocentesco della raccolta di testi poetici ordinati per generi metrici, utilizzato talvolta anche per le rime dell'Aquilano, ha il chiaro intento di mostrare come l'aspirante poeta-cortigiano sappia già manipolare non solo forme, ma anche $i$ materiali della tradizione lirica del secolo precedente derivati dalle opere di Poliziano, Cariteo e, qua e là, anche di Sannazaro.

Di ben altro tenore e complessità appare invece la Catrina di un forse - e sottolineo forse - ancor giovane Francesco Berni. Questo atto scenico rusticale edito postumo, dapprima nel 1567 e di nuovo intorno al $1600^{5}$ con un forse mutilo Mogliazzo di dubbia attribuzione, si ispira cbia- 
ramente al genere nenciale e più in generale alla produzione poetica di ambito laurenziano, il cui esempio resta vivissimo e attivo ancora per tutto il Cinquecento nella produzione meno frequentata e purtuttavia abbondantissima di commedie rusticali di matrice toscana, una produzione su cui avrò modo di tornare in seguito. Nell'operetta in ottave, le cui sorti editoriali appaiono ancora oggi quanto meno complesse, alla tradizionale tensione linguistica tra villani, o tra villani e cittadini, fanno eco un sapiente gioco di doppi sensi e di non-sensi e un procedere per botta e risposta che lascerebbe presagire una non impossibile recitazione della composizione, caso mai in ambito 'riservato'. Ora, che un certa leggerezza di toni anche a carattere sessuale albergasse nella produzione nenciale e villanesca in genere è cosa risaputa, ma nella Catrina il rafforzamento e talvolta la distorsione di tale carattere finisce per manomettere seriamente il canone preesistente. Com'è noto, la poesia del Berni intraprese ben altri percorsi, ma del raffinatissimo sperimentalismo burlesco della stagione più alta si rintracciano qua e là degli scampoli anche nel tratto anti-accademico della Catrina, che pare presagire a più radicali evoluzione in senso antinormativo e basso della maniera bucolica. Uno degli autori più rappresentativi di questa evoluzione è Giovanni Agostino Caccia, di cui Giuseppina Stella Galbiati ha saggiamente evocato il carattere novatore e più spiccatamente satirico. ${ }^{6}$ In opere come l'Erbusto e la commediola intitolata Filena, ${ }^{7}$ infatti, il ricorso alla forma ormai rodata dell'egloga rappresentativa accentua il valore eversivo dell'esperimento al contempo dissacrante e coltissimo del Caccia. Questi reimpiega metri, topoi e modelli aulici (Petrarca in primis) per mettere in luce in modo antifrastico le brutture di questo basso mondo, non di rado in chiave apertamente comicosatirica. Insomma, in modo forse paradossale, o quanto meno inaspettato, il Caccia prende di mira in una commedia pastorale gli stessi stereotipi sociali e letterari messi alla berlina nei suoi capitoli ${ }^{8}$ e nelle scritture anti-auliche coeve di autori quali il Berni, il Bentivoglio e l'Aretino. Per far ciò, l'autore accentua il tono basso e realistico del dettato, il ricorso alla metafora sessuale, di contro ad un estenuante ripresa del linguaggio aulico, mentre il contesto mitologico e antichizzante diviene sempre meno percepibile. Che tale abbassamento del registro, fin quasi a sfiorare il grottesco, sia il frutto consapevole di una messa in discussione dell'ormai frusta tradizione pastorale tardoquattrocentesca, è evidente fin dal prologo dell'Erbusto, che recita: 
Venga il cancaro a Lacrito e a chi gli insegnò far versi! Oh questa è bella! Io non so pur cominciare: non mi sarei ma creduto d'aver sì poco cervello! [...] A pena mi sovviene dei nomi, benché un certo pastore innamorato si chiama (s'io non m'inganno) "Vo' tu ch'io tel metta", no, no: Ameto! Un altro Erbosto, Erbasto, Erbusto cred'io, e la donna Fora, o sia Dentro, per dir meglio. ${ }^{9}$

Ovviamente la donna in questione si chiama Flora e, nonostante la delicatezza evocatrice del nome, ha maniere alquanto spicce ed un lingua pronta, poco avvezza alle ricercatezze liriche di certe egloghe coeve di matrice colta e antichizzante.

Prove di questo tipo escludono d'emblée una destinazione encomiastica tipica di quel cospicuo lotto di egloghe diffuse in occasione di matrimoni o cerimonie funebri, e ancor meno un impiego a scopo celebrativo e allegorico, fatto salvo - forse - per alcuni testi piscatori di Andrea Calmo, il quale coniuga invenzione metaforica, pratica attoriale, diversi registri linguistici e una calibrata irriverenza che muove al sorriso anche nell'egloga in onore della Serenissima il cui incipit recita $\mathrm{O}$ donzelletta che in le acque insalae. ${ }^{\text {Io }} E$ pensare che la ricezione dell'egloga in Francia è, nel XVI secolo, quasi sempre di matrice encomiastica e occasionale, mai giocosa e satirica, rarissimamente a carattere moraleggiante e allegorico. Che si vedano, a tale proposito la prima traduzione delle bucoliche Spagnoli (IS3I), " ' ma soprattutto le egloghe di Clément Marot in morte di Louise de Savoie (I534), ${ }^{\mathrm{I}}$ la piscatoria d'Hugues Salel in morte di François de Valois (I536), "3 la Saulsaye. Eglogue de la vie solitaire di Maurice Scève o ancora l'omaggio nunziale di François Habert a Filippo II e Elisabetta di Valois (I559). ${ }^{4}$ Si noti inoltre che, negli anni Sessanta, in Francia non mancarono dei détournements dell'egloga in senso confessionale in alcuni libelli propagandistici riformati, sebbene il genere si presti assai poco all'esercizio in questione. ${ }^{\mathrm{I}}$ Per $i$ lazzi scenici facilmente spendibili in tipografia, in Francia, si ricorreva infatti alle cosiddette farces $e$ dialogues plaisants. ${ }^{\text {I6 }}$ D'altronde, anche nella penisola, non pochi poligrafi e poeti comico-satirici di estrazione colta della metà del secolo XVI si dedicarono soprattutto alla redarione di egloghe di tipo celebrativo; basti pensare ai componimenti del Lasca in onore delle nozze di Cosimo I o in morte di una gentildonna fiorentina, ${ }^{\mathrm{I}}{ }^{\text {e perfino il Doni, nel Ragio- }}$ namento di diversi affanni umani figurante nei suoi Marmi, offre 
un esempio non spregevole di una Pastorale in terza rima in morte «del suo signore». ${ }^{18}$

Un tutt'altro tipo di sperimentalismo, sempre di matrice comica e di ascendenza attoriale come quello del Calmo, ma con insospettati risvolti editoriali, è quello praticato nei primi decenni del XVI secolo dallo Strascino che, per la sua unicità e per le suggestioni che esercitò sulla produzione senese successiva, e più generalmente dell'Italia centrosettentrionale, meriterebbe una più lunga disanima. ${ }^{19}$ La commedia rusticale intitolata Coltellino è un chiaro esempio di come la tradizione toscana della satira del villano abbia potuto integrare, trasformandole, le scritture bucoliche d'area padana. Se la critica più recente tende a riabilitare non solo da un punto di vista storico-culturale, ma anche squisitamente letterario, opere di questo tipo, appare difficile per il moderno lettore comprendere la robusta e prolungata fortuna di questa pièce di cui si conoscono numerosi adattamenti e che servi da modello editoriale ad una moltitudine di opere simili, spesso di autori pressoché sconosciuti o volontariamente anonimi, che mettono in scena e per iscritto villani adusi a scimmiottare $i$ patimenti amorosi di arcadici pastorelli con esiti solo sporadicamente comici. Siamo lontanissimi dalla stralunata e affascinante alterità dei personaggi ruzantiani, anche perché il testo a stampa del Coltellino dello Strascino occulta inevitabilmente la dimensione mimica e gestuale della rappresentazione, il che riduce significatamente lo spessore drammatico dei personaggi. Che ci piaccia o meno, delle farsesche vicende di Berna che tenta di suicidarsi con un inoffensivo coltellino troveremo traccia anche nella produzione comica dei Rozzi a distanza di vari decenni dalla scomparsa dell'autore. Quello che soprattutto mi preme sottolineare in questa sede è come, nel corso del Cinquecento, muti sensibilmente il concatenarsi delle diverse fasi di produqione e divulgazione dei testi comici a carattere bucolico secondo lo schema: redazione (individuale o collettiva), recitazione (eventuale), diffusione a stampa.

Quel che intendo dire è in sostanza che, dati alla mano, a partire dal quarto decennio del Cinquecento la fortuna della bucolica rusticale, così come quella di egloghe di ogni tipo e di altri scritti occasionali di matrice pastorale, sia sempre meno ancorata alla declamazione e alla circolazione cittadina, eventualmente di ambito cortigiano o accademico, $e$ sempre più legata ad una fruizione individuale e "decentrata" di testi che, spesso, nascono per essere esclusivamente letti e non interpretati. 
Da un rapido excursus dei principali cataloghi italiani e stranieri si possono estrarre una serie di dati che, sebbene in modo approssimativo, illustrano meglio quanto appena evocato. In questa occasione mi limito a riportare quanto si evince dal catalogo EDITI6, che sebbene non esaustivo, fornisce un ottimo punto di partenza, tanto dal punto di vista quantitativo che qualitativo, per una analisi che potrà essere approfondita in seguito. Una volta operate le debite scremature e qualche aggiustamento alla lunga lista di risultati ottenuti grazie a delle ricerche per parola chiave, possiamo rilevare per esempio che, durante l'intero Cinquecento, vennero pubblicate almeno 350 edizioni recanti nel titolo il termine egloga/egloghe, al netto degli adattamenti virgiliani. Solo una piccola parte di tali edizioni consta di raccolte di rime o di "opere nove" in cui l'egloga figura quale uno dei vari generi frequentati dagli autori. Più spesso siamo in presenza di opuscoli in-octavo di pochi quaderni che diffondono singole egloghe o raccolte di pochi componimenti recanti, non di rado, un frontespizio illustrato. Le 350 edizioni sono ascrivibili a $7 I$ autori noti e meno noti, 52 opuscoli escono in forma anonima, e sussistono almeno 7 edizioni di raccolte poetiche di più autori.

Anche l'indicazione dei luoghi di edizione fornisce qualche informazione interessante: se i poli editoriali che la fanno da padrone sono, per ovvie ragioni, Siena e Venezia con decine e decine di edizioni, altri centri di produzione di egloghe di ogni sorta sono, in ordine decrescente per importanza, Milano, Firenze, Bologna e Napoli. La diffusione capillare in territori più decentrati è assicurata da un buon numero di tipografie locali. La mappatura territoriale è per il momento approssimativa, come dicevo, ma si possono già rilevare alcune aree di produzione/diffusione assai interessanti. Si veda ad esempio questa tabella:

Piemonte:

Trino I ed.

Carmagnola I ed.

Entroterra veneto:

Verona 7 ed.

Padova I ed.

Treviso I ed.

Vicenza I ed. 
Area lombarda e padana:

Pavia 3 ed.

Piacenza 2 ed.

Ferrara 2 ed.

Brescia I ed.

Lazio e Italia centrale:

Roma s o 6 ed.

Perugia 4 ed.

Osimo 3 ed.

Rimini 2 ed.

Urbino 2 ed.

Fano I ed.

Fermo i ed.

Orvieto I ed.

Ascoli I ed.

Sicilia:

Palermo i ed.

Oltre alle città in cui erano attive università, accademie e cenacoli letterari, $i$ dati fin qui raccolti testimoniano di una diffusione di tali opuscoli in un'area umbro-marchigiana assai estesa, mentre è da sottolineare la rarità di testi in area piemontese e ligure.

Tra gli autori che incontrano maggiormente il favore del pubblico e dei librai figurano il senese Pierantonio Legacci ${ }^{20}$ con una cinquantina di edizioni, l'A quilano secondo la tradizione tramandata dalle raccolte postume e - spesso - posticce (almeno una trentina di edizioni), il Tebaldeo con 22 edizioni, Leonardo Maestrelli con is e Notturno Napoletano con I4 edizioni. Si noti inoltre che le egloghe del fantomatico Notturno escono sempre al centro-nord (da Perugia in su, perintenderci) $e$ che il computo in questione non tiene conto delle numerose ristampe dell'egloga pastorale Lilia, uscita senza alcuna menzione dell' autore una miriade di volte con titoli talvolta diversi. I luoghi di edizione delle operette anonime restituiscono lo stesso paesaggio editoriale: se Venezia e Siena restano i maggiori centri di produzione, non mancano qua e là dei rarissimi opuscoli editi in città periferiche, come nel caso dell'unicum Egloga pastorale in satira. Intitolata Il piaceuole flagello delle donne, pubblicata a Orvieto, presso Rosato Tintinnassi, nel is82. 
Mi son divertita a ripetere il medesimo esercizio per stringhe testuali che permettono di valutare approssimativamente il numero di commedie o comedie rusticali, pastorali o villanesche (facendo inevitabilmente di tutt'un'erba un fascio) ed ecco qui il risultato. Sussistono a tutt'oggi ro\& edizioni, di cui 6 anonime, redatte da almeno 24 autori diversi, e cosi suddivise, $49 \mathrm{com}(\mathrm{m})$ edie rusticali, $59 \mathrm{com}(\mathrm{m})$ edie pastoriali.

Le commedie rusticali sono principalmente edite in Toscana, a Firenze e Siena, più raramente a Venezia e in almeno un caso a Roma. Le commedie pastorali sono sempre pubblicate in gran numero a Firenze e Siena, ma anche a Torino, Parma, Ancona, Orvieto, Roma, Macerata, Ferrara, Fano, il che ci conferma un dato ben noto ed evocato precedentemente, cioè la matrice autenticamente toscana di molta letteratura comica rusticale di ascendenza laurenziana e boccacciana e una minore uniformità di provenienze e fruizione per i generi più marcatamente 'pastorali'.

Molti autori toscani si cimentano anche in commedie villanesche almeno 36 - spesso difficilmente distinguibili dalle rusticali per temi, modi e meccanismi retorici, tant'è che il più delle volte l'aggettivo villanesco è sinonimo puro e semplice di rusticale. Al suddetto lotto di commedie (ormai abbiamo raggiunto $i$ I45 specimina) si aggiungono almeno 47 commedie che arborano, sin dal titolo, la presenza di contadini e villani, il che porta il nostro computo a circa 200 edizioni, essenzialmente di provenienza toscana, ad opera di autori ed editori che coprono tutti i decenni del XVI secolo.

Se i principali autori di egloghe, quelli insomma che producono dei veri best-sellers del genere, provengono dallintera penisola (si pensi al Legacci, al Tebaldeo e a Notturno Napoletano), quanti si cimentano in commedie rusticali e villanesche son tutti di provenienza toscana (Bastiano di Francesco, Ascanio Cacciaconti, Niccolò Campani, Angelo Cenni, il Fumoso, Francesco Mariano Trinci, ecc.), mentre appare regionalmente più variegato il lotto di coloro che redigono favole pastorali in forma di commedia. Si noti inoltre che i cosiddetti "irregolari", che di scrittura comico-burlesca e drammaturgica se ne intendevano e si erano già cimentati in scritture bucoliche (cioè Aretino, Berni, Bentivoglio, Caccia, Lasca, ecc.), sono assolutamente assenti dallo scenario che ho tentato di tracciare sino a qui. La loro produzione comica e teatrale sceglie, com'è noto, più colti e 'autorizzati' contesti, vesti editoriali più 
dignitose e accurate, e si pone su un altro livello cultuale e merceologico. Insomma, i ludi dei Rozzi ben poco banno a che spartire con quelli degli accademici Infiammati e Veneziani, altri $i$ modelli che presiedono all'ispirazione poetica, altri $i$ destinatari e $i$ canali di diffusione. ${ }^{2 \mathrm{I}}$

La veste editoriale di egloghe comiche e commedie rusticali tradiscono, fin dal frontespizio, ambizioni più modeste rispetto alle opere celebrative, allegoriche e 'all' antica'. Si vedano, a titolo d'esempio, le riproduzioni dei frontespizi di La Agia (ed. I552), un'egloga celebrativa di Luca Contile in onore di Ippolita Gonzaga, e la commedia pastorale Aretusa del Lollio (ed. I564). ${ }^{22}$ In entrambi i casi la ricercatezza tipografica e l'eleganza della composizione della pagina è conforme alla natura dell'opera e al prestigio di autori e destinatari. I frontespizi di egloghe rappresentative a carattere comico testimoniano invece di piu modeste ambizioni, come nel caso dell'egloga di Maggio del mugellano Maestrelli o dell'egloga di Mitio e Achario di Notturno Napoletano. ${ }^{23} \dot{E}$ evidente, in entrambi $i$ casi, che tale prodotto editoriale è pensato per un pubblico piu vasto e sovente meno abbiente, il che consente il reimpiego di serie di caratteri d'ogni genere (gotici, semi-gotici, romani) e di decori tipografici di scarsa qualità, un pubblico che si lascia sedurre più facilmente da un'illustrazione maldestra che da una chiara e areata composizione. Vero è che per queste operette di poco prezzo, ristampate numerose volte, spesso si tende ad investire più nella xilografia di apertura che nella qualità di carta e caratteri, soprattutto per le prime edizioni, quando il testo cerca ancora il successo che - forse - merita; per le ristampe posteriori, quando ormai l'opera è diventate un bestseller, ci si può accontentare di semplici decori tipografici di reimpiego, tanto più che sovente si è persa persino la memoria del soggetto che decorava le prime stampe. Certi legni risultano cosi usati fino all'osso, come nel caso delle ristampe dell'anonima Commedia dei due contadini Beco e Fello, ${ }^{24}$ mentre per le ciarle di Tonio e Pippo nella commedia omonima si è dovuto procedere alla riproduzione (ivi compreso a contrario) della xilografia originale almeno due volte. ${ }^{25}$ Per questo tipo di edizioni si utilizzano spesso materiali che ben poco banno a che vedere con il contenuto del testo, come nel caso della Discordia d'Amore del Fumoso senese, ${ }^{26}$ mentre quando si vuole accentuare la natura dialogica e performativa del componimento, non si esita a far produrre delle vignette recanti i nomi dei personaggi (Egloga rusticale di Niccola). ${ }^{27}$ In altri casi, il soggetto iconografico si concentra su 
un momento saliente dell'azione comica, come nel caso dell'egloga di Tognino che impegnò la moglie, oppure sullo stratagemma narrativo che anima l'intera pièce (per esempio nell'egloga di Mecoccio che è alla ricerca del proprio cuore). ${ }^{28}$ Si noti inoltre che certe incisioni "all'antica" o a carattere mitologico vengono utilizzati per commedie bucoliche diverse, sebbene $i$ messaggi veicolati rispettivamente dal testo e dall'immagine risultino discordanti. Ritroviamo infatti la medesima illustrazione nell'Egloga rusticale di Michelagnolo e nelle due edizioni della più nota commedia di Bastiano di Francesco intitolata Vellera. ${ }^{29}$ Quando si è in presenza di un vero e proprio successo editoriale, come il celebre Coltellino dello Strascino, si finisce per raschiare $i$ fondi della cassetta del compositore e riesumare materiali tipografici che davvero han poco a che vedere soggetto in questione. ${ }^{30}$ Qua e là spuntano anche personaggi mitologici e contesti arcadici che restano tutto sommato sporadici.

Quanto detto sin qui mi induce a credere che gran parte della produzione di egloghe e altre forme bucoliche a carattere 'perfomativo' abbiano, strada facendo, perduto la loro primigenia caratteristica, cioè quella declamativa e drammaturgica, per diventare un oggetto letterario e tipografico autonomo, destinato a un tutt'altro tipo di fruizione. Se alcune delle opere menzionate son germinate all'interno di accademie e cenacoli più $i$ meno colti, gran parte di esse hanno intrapreso percorsi davvero stravaganti, finendo sotto $i$ torchi di stampatori di provincia spesso svariati decenni dopo la morte degli autori. Ciò ha permesso il fiorire di una rinnovata produzione comica locale anche in luoghi eccentrici rispetto alle grandi vie di circolazione di modelli e pratiche culturali. Tale fenomeno ba finito per influenzare altri generi poetici e drammaturgici coevi; qualche anno fa, in occasione della pubblicazione di un lotto di libretti di ispirazione pasquinesca, mi son trovata tra le mani una commedia anonima a carattere occasionale e di matrice politica edita nel I522, a Perugia, dai fratelli Cartolari. ${ }^{3}$ La commedia in questione mette in scena personaggi storici, allegorici e mitologici in un succedersi di strutture metriche eterogenee al solo intento di commentare l'attualità politica italiana ed europea da un punto di vista decentrato, quello della città umbra, seppure in linea con le strategie diplomatiche messe in atto dalla Serenissima. Il testo in questione risente fortemente dellimpianto bucolico di molte commedie coeve, ma su di esso si innesta parzialmente la nascente tradizione pasquinesca in vernacolo e le sug- 
gestioni esercitate da componimenti occasionali a carattere squisitamente politico. Di certo, l'anonima Comedia perugina non è il solo esempio di un sincretismo riscontrabile in area centro-italiana ed ulteriori investigazioni nei fondi e nei cataloghi nazionali e internazionali permetteranno di chiarire meglio come la diffusione a stampa di egloghe rappresentative e commedie rusticali concepite per un consumo tutto sommato locale e occasionale possa aver influito sulla poligenesi di forme comiche ibride e sperimentali almeno fino agli anni Sessanta del Cinquecento.

Chiara Lastraioli 
I. Mutuo l'espressione dal titolo del prezioso volume Cinquecento capriccioso $e$ irregolare. Eresie letterarie nell'Italia del Classicismo, a cura di P. Procaccioli e A. Romano, Manziana, Vecchiarelli, I999.

2. Opera noua zoe strambotti sonetti capitoli epistole barzellette \& una desperata (Impresso in Venetia per Nicolo Zopino, i 5 I 2 adi XXII de zenaro). Sull'egloga della prima metà del secolo XVI si veda soprattutto L. Borsetto, L'egloga in sciolti nella prima meta del Cinquecento: appunti sul Liber di Girolamo Muгio, Roma-Padova, Antenore, 2004 e F. Bortoletti, Egloga e spettacolo nel primo Rinascimento: da Firenze alle corti, Roma, Bulzoni, 2008. Utili anche gli studi di P. Cosentino, Una "zampogna Tosca" alla corte di Francia: le egloghe in versi sciolti di Luigi Alamanni, in "Filologia e critica», I, 2003, pp. 70-95 e F. Venturi, Bernardino Baldi e la tradizione dell'egloga nel Cinquecento, in «Giornale Storico della Letteratura Italiana», 622, 201 I, pp. 249-58.

3. Ora in Pietro Aretino, Poesie varie, a cura di G. Aquilecchia e A. Romano, vol. I, Roma, Salerno, I992, p. 39.

4. Sulla produzione di questi autori si rinvia a A. Rossi, Serafino Aquilao e la poesia cortigiana, Brescia, Morcellianna, I980; C. A. Addesso, 'Portano i latron pieni i lor saini: elementi della teatralità aragonese nella produðione egloghistica e farsesca di Serafino Aquilano e Antonio Ricco, in «Rivista di letteratura teatrale», 6, 2013, pp. 9-22; F. Gavagni, L'Unico Aretino e la Corte dei Duchi d'Urbino, Arezzo, Gagliani, I9o6; D. Gnoli, La Roma di Leone X, Milano, Hoepli i938, pp. 266-99.

5. La Catrina. Atto scenico rusticale di m. Francesco Berni, in Fiorenza, appresso Valente Panizi e compagni, i 567 et La Catrina, atto scenico rusticale di m. Francesco Berni, insieme col frammesso detto il Mogliazzo, s.l.n.d. [I60o?].

6. G. S. Galbiati, Un poeta satirico del Cinquecento, Giovanni Agostino Caccia, Pisa, Giardini, I991.

7. Le due egloghe sono oggi in Giovanni Agostino Caccia, Rime (I546), a cura di B. Buono, Milano, Lampi di Stampa, 20 ı.

8. Cfr. Giovanni Agostino Caccia, Satire, et Capitoli piacevoli (I549), con un'appendice di testi inediti di Bartolomeo Taegio, a cura di B. Buono, Vignate, Lampi di stampa, 2013.

9. CACCIA, Rime (I546), p. 227.

io. Si veda a tale proposito Andrea Calmo, Le bizzarre, faconde et ingegnose rime pescatorie, testo e commento a cura di G. Belloni, Venezia, Marsilio, 2003, [Pescatoria VI], pp. I 24-26.

I I. Les bucoliques de Frère Baptiste Mantuan, nouvellement traduictes de latin en rigme francoyse par Michel d'Amboyse, aultrement dict l'Esclave fortunay, [...] lesquelles sont divisées en dix églogues et nouvellement imprimées à Paris, Paris, [Alain Lotrian et Denis Janot, I $53 \mathrm{I}]$, in $-4^{\circ}$.

i 2. Clément Marot, La Suite de l'Adolescence clémentine, dont le contenu sensuyt: une Églogue sur le trespas de Madame Loyse de Savoye, mère du Roy, les Élégies de l'autheur, les Epistres différentes, les Chantz divers, le Cimetière et le Menu, Dictier au conte de nasso [par 
J. Molinet], l'Ordonnance perspective salutaire. Moralles paradoxes du Lys, on les vend à Lyon, en la rue Mercière, à la Fleur de lys d'or, en la boutique de Guillaume Boulle, I 534 , in-1 $6^{\circ}$.

I 3. Hugues SAlel, Eglogue marine sur le trespas de feu M. Francoys de Valoys, daulphin de Viennois, filz aisné du Roy, ensemble ung chant royal sur l'entreprinse de l'Empereur contre le Roy, et honteuse fuite dudict Empereur. Et autres choses. Le tout composé par Hugues Salel, de Quercy, Paris, impr. d’O. Maillard, I 536 , in $-8^{\circ}$.

I4. FRANÇOIS HABERT, Églogue pastorale sur l'union muptiale de treshault et très puissant seigneur Philippes, roy d'Hespagne, et de madame Élisabeth, première fille du roy Henri II, Paris, J. Moreau, I 5 59, in- $8^{\circ}$.

I 5 . Si veda per esempio l'Eglogue de deux bergers, demonstrant comme la ville de Lyon a esté réduite à la religion vrayement chrestienne, par la pure prédication de l'Evangile, à Lyon, par Jean Saugrain, 1564 , in- $8^{\circ}$.

16. Cfr. B. Rey-Flaud, La farce ou La machine à rire. Théorie d'un genre dramatique, I450-I550, Genève, Droz, I984, e A. Tissier, La farce en France de I450 à I550: recueil de textes établis sur les originaux, présentés et annotés, Paris, Editions Sedes/CDU, 1976 et I $98 \mathrm{I}$.

i7. Cfr. Anton Francesco Grazzini detto il Lasca, Egloghe ed altre rime, ora per la prima volta accuratamente pubblicate, Livorno, Domenico Poggiali, I799. Sulla produzione del Lasca si veda soprattutto M. Plaisance, Antonfrancesco Grazzini dit Lasca, I505-1584. Ecrire dans la Florence des Médicis, Manziana, Vecchiarelli, 2005.

18. Cfr. Anton Francesco Doni, La terza parte de marmi, del Doni fiorentino; allo illustrissimo, \& eccellentissimo signore, il signor don Ferrante Gonzaga dedicati, in Vinegia, per Francesco Marcolini, I 5 52, pp. 61-62.

I9. Sulla produzione dello Strascino si veda soprattutto Niccolò Campani, Lo Strascino da Siena e la sua opera poetica e teatrale, a cura di M. Pieri, Pisa, ETS, 20 Io; C. Valenti, Comici artigiani. Mestiere e forme dello spettacolo a Siena nella prima metà del Cinquecento, Modena, Panini, I 992 e Ead., Le egloghe rusticali dello Strascino e la multiformità attorica. In margine al 'teatro popolare', in «Quaderni di teatro», v, I982, pp. 56-68.

20. Alcuni testi del Legacci figurano in Teatro comico del Cinquecento. La tonaca in commedia, a cura di S. Termanini e R. Trovato; testi di Pierantonio Stricca Legacci, Luca Contile, Cesare Odoni... [et al.], Torino, Unione tipograficoeditrice torinese, 2005.

2 I. Sulla produzione dei Rozzi si vedano, tra gli altri studi, R. Alonge, Il teatro dei Rozzi di Siena, Firenze, Olschki, 1967 e L. Riccò, "Su le carte e fra le scene". Tetro in forma di libro nel Cinquecento italiano, Roma, Bulzoni, 2008.

22. Si vedano su EDITi 6 i frontespizi di LuCa Contile, La Agia. Egloga del Contile recitata da la sopra humana donna Hipolita Gonzaga, e da molte altre sue gentildonne (Excudebant Mediolani, Valerius et Hieronymus Metii, i 5 2), CNCE I 3 180, e 
Alberto Lollio, Aretusa comedia pastorale [...], In Ferrara, per Valente Panizza mantoano stampator ducale, I 564 CNCE 28342. Sul Contile si veda soprattutto Luca Contile da Cetona all'Europa: atti del seminario di studi, Cetona 20-2 I ottobre 2007, a cura di R. Gigliucci, Manziana, Vecchiarelli, 2009 e E. Pietrobon, Per una rilettura delle Rime di Messer Luca Contile, in «Italique», I7, 20I4, pp. 207-27.

23. Cfr. L. Maestrelli, Egloga pastorale di maggio, Composta per Lionar. di ser Ambrogio alias Mescolino interlocvtori Aurorio, Frolio, Gaudio, \& Damasia, s.l.n.d. [circa I 520 ], CNCE 63538, e Notturno Napoletano, Egloga de Notturno Neapolitano interloquutori Mitio et Achario, s.l.n.d., CNCE 61780. Si noti inoltre che la Fondation Barbier Mueller pour l'étude de la poésie italienne à la Renaissance possiede un'altra opera di Mescolino Maestrelli, l'Egloga rusticale del Targone, Stampata in Siena per Francesco di Simione. Ad ista[n]tia di Giouan[n]i di Alisandro Libraro Adi 23 di Noue[m]/bre. M.D.XXXXII (cote XXXX).

24. Cfr. le tre edizioni della Commedia di dua contadini intitolata Beco \& Fello, forse tutte fiorentine, stampate tra il i 550 e il I 580 : I) Commedia di dua contadini intitolata Beco et Fello, In Firenze, I 568 , in- $8^{\circ}$, CNCE I 4963; 2) [idem], In Firenze, presso il Vescouado, I 562 , in- $8^{\circ}$, CNCE I 4962; 3) [idem], s.l.n.t. [1 s...], in-8 $8^{\circ}$, CNCE I 496 I.

25. Cfr. le tre edizioni della Commedia Commedia di Tonio e Pippo [...], forse tutte e tre fiorentine della metà del Cinquecento: I) Commedia di Tonio, e Pippo contadini, \& l'hoste, s.l.n.t., [1 5 ..], in- $8^{\circ}$, CNCE $1497 \mathrm{I}$; 2) Commedia di Tonio, e Pippo contadini \& l'host [e], s.l.n.t., [I ...], in- $8^{\circ}$, CNCE I 4970; 3) Comedia di Tonio e Pippo contadini \& lhoste, [Firenze], fece stampare Bartholomeo di Mattheo Castelli, in- $8^{\circ}$, CNCE I 4939 .

26. Fumoso, Discordia d'amore commedia nuona rusticale [...], (In Siena, per Francescho di Simeone, ad istantia di Giouanni d'Alisandro libraro, il di 3 I di maggio I 550 ), CNCE $6902 \mathrm{I}$.

27. Pierantonio Legacci, Egloga rusticale di Niccola. Composta per Pierantonio dello Stricha Legacci, (Stampata in Siena, per Michelagnolo di Bernardino, ad instantia di Giouanni di Alixandro libraio a di primo di octobre i 533 ), CNCE 63015.

28. Cfr. Pierantonio Legacci, Egloga rusticale di Tognino del Cresta che impegno la moglie. (Stampata in Siena, per Michelagnolo di Bernardino Castagni, ad istantia di Giovanni di Alixandro libraio, adi VIIII di aprile i 533 ), CNCE 63 I 36 e PIERANTONIO LeGACCI, Egloga rusticale di Mecoccio, che a perso el cuore, e vallo cercando. (In Siena, per Ant. Mazochi Cre., ad instantia de Giouanni d'Alex. lib., I 544), CNCE I 8046.

29. Cfr. Egloga rusticale di Michelagnolo. Interlocutori Michelagnolo cittadino, Balestro, Tachone villani, Gieca moglie di Taccone \& Bargello. - [ I ...], CNCE 39554; BASTIANO DI Francesco, Vallera commedia nuona pastorale, et rusticale molto sententiosa, et copiosa di esempli, et istorie poetiche [...], In Siena, I 546 (Nuouamente stampata in Siena, presso a san Viglio, adi X di ottobre I 546), CNCE 62764.

30. Si vedano su EDITi6 i frontespizi delle tre edizioni che seguono della stessa egloga alla villanesca (o commedia rusticale) dello Strascino comunemente nota come Il Coltellino: I) Coltellino commedia rusticale. Composta per Niccolò Campana alias 
Strascino, s.l., [non prima del I 520 ]., in- $8^{\circ}$, CNCE 8787; 2). Egroga [!] villanesca del poeta Strascino. Interlocutori in prima vno anutia cioe dice il prolagho \& chome glie uno uillano che unole intrare che si chiama el Berna e di poi entra dentro Berna \& inchomincia il primo di poi parlla con Togna ninpha ho pastora Taphano Ellenzo. La commedia del coltellino, (In Siena, per Simione di Niccolo cartolaro, adi 5 de febraro I 520 ), CNCE 8785 ; 3) Coltellino comedia rusticale. Composta per Niccolo Campani sanese, In Siena, [Luca Bonetti], I 57 I, in- $8^{\circ}$, CNCE 8799.

3 I. Comedia. Interlocutori: Italico Pasquin: Suffragio Theutonico et Galico Vinetia Fortuna Discordia Fama Virtu Tempo Clotho Lachesis Antropos nouamente recitata in Perosa., s.l.n.d., in- $8^{\circ}$, CNCE I 2862 . Ora, ad opera di chi scrive, in Pasquinate, grillate, pelate et altro Cinquecento librario minore, Manziana, Vecchiarelli, $20 \mathrm{I} 2$. 